\title{
ARTICLE
}

Received 14 Jun 2014 | Accepted 19 Sep 2014 | Published 30 Oct $2014 \quad$ DOl: 10.1038/ncomms6352

\section{A system for the continuous directed evolution of proteases rapidly reveals drug-resistance mutations}

\author{
Bryan C. Dickinson ${ }^{1, \star, \dagger}$, Michael S. Packer ${ }^{1, \star}$, Ahmed H. Badran ${ }^{1} \&$ David R. Liu ${ }^{1,2}$
}

The laboratory evolution of protease enzymes has the potential to generate proteases with therapeutically relevant specificities and to assess the vulnerability of protease inhibitor drug candidates to the evolution of drug resistance. Here we describe a system for the continuous directed evolution of proteases using phage-assisted continuous evolution (PACE) that links the proteolysis of a target peptide to phage propagation through a protease-activated RNA polymerase (PA-RNAP). We use protease PACE in the presence of danoprevir or asunaprevir, two hepatitis $\mathrm{C}$ virus ( $\mathrm{HCV}$ ) protease inhibitor drug candidates in clinical trials, to continuously evolve HCV protease variants that exhibit up to 30-fold drug resistance in only 1 to 3 days of PACE. The predominant mutations evolved during PACE are mutations observed to arise in human patients treated with danoprevir or asunaprevir, demonstrating that protease PACE can rapidly identify the vulnerabilities of drug candidates to the evolution of clinically relevant drug resistance.

\footnotetext{
${ }^{1}$ Department of Chemistry and Chemical Biology, Harvard University, 12 Oxford St, Cambridge, Massachusetts 02138, USA. ${ }^{2}$ Howard Hughes Medical Institute, Harvard University, 12 Oxford St, Cambridge, Massachusetts 02138, USA. * These authors contributed equally to this work. $\dagger$ Present address: Department of Chemistry, The University of Chicago, 929 E. 57th St., Chicago, Illinois 60637, USA. Correspondence and requests for materials should be addressed to D.R.L. (email: drliu@fas.harvard.edu).
} 
A mong the more than 600 naturally occurring proteases that have been described ${ }^{1}$ are enzymes that have proven to be important catalysts of industrial processes, essential tools for proteome analysis and life-saving pharmaceuticals ${ }^{2-5}$. Recombinant human proteases including thrombin, factor VIIa, and tissue plasminogen activator are widely used drugs for the treatment of blood clotting diseases ${ }^{4}$. In addition, the potential of protease-based therapeutics to address disease in a manner analogous to that of antibody drugs ${ }^{6,7}$, but with catalytic turnover, has been recognized for several decades ${ }^{4,8}$. Natural proteases, however, typically target only a narrowly defined set of substrates, limiting their therapeutic potential. The directed evolution of proteases in principle could generate enzymes with tailor-made specificities, but laboratory-evolved proteases are frequently non-specific, weakly active or only modestly altered in their substrate specificity, limiting their utility ${ }^{9-14}$.

In addition to their importance as current and future therapeutic agents, proteases have also proven to be major drug targets for diseases including cardiovascular illness, infectious disease and cancer ${ }^{15,16}$. While drug specificity and potency are characterized and optimized during pre-clinical studies, the evolution of drug resistance is often not well understood until it arises in patients, despite the strong relationship between drug resistance vulnerability and a lack of therapeutic efficacy. For example, resistance to HIV and HCV protease inhibitors can arise in as few as 2 days of clinical use ${ }^{17}$ and frequently leads to viral rebound and poor treatment outcomes ${ }^{18-21}$. The speed with which drug resistance can arise in the clinic endangers patients and puts years of drug development efforts before such a determination at risk. Unfortunately, characterizing the potential of protease inhibitors to be overcome by the evolution of drug resistance using methods such as mammalian cell culture, animal models or yeast display-based laboratory evolution is time- and labor-intensive ${ }^{22,23}$. As a result, identifying drug resistance vulnerabilities of early-stage preclinical candidates is not a common practice.

Phage-assisted continuous evolution (PACE) in principle could serve as a rapid, high-throughput method to evolve protease enzymes and to reveal resistance to protease inhibitor drug candidates, analogous to previous uses of stepwise protein evolution to study antibiotic resistance ${ }^{24}$. During PACE, continuously replicating M13 bacteriophage in a fixed-volume vessel (a 'lagoon') carries an evolving gene of interest. Phage with genes encoding proteins with the desired target activity preferentially replicate because target activity triggers the production of pIII, an essential component in the bacteriophage life cycle ${ }^{25}$. Because the lagoon is continuously diluted by a constant influx of host $E$. coli cells, phage encoding inactive variants produce non-infectious progeny that are rapidly diluted out of the lagoon. Dilution occurs faster than cell division but slower than phage replication, ensuring that mutations only accumulate in the phage genome. Because evolution during PACE takes place continuously without researcher intervention, hundreds of theoretical rounds of evolution can be performed per week. We speculated that PACE could be well suited to the directed evolution of proteases, which may require many successive mutations to remodel complex networks of contacts with polypeptide substrates ${ }^{26,27}$. Moreover, the speed of PACE may enable the rapid identification of mutations that confer resistance to protease inhibitors. To date, however, PACE has only been reported to evolve RNA polymerase enzymes ${ }^{25,28-30}$.

Here we describe the development and application of a system for the continuous directed evolution of proteases. This system uses an engineered protease-activated RNA polymerase (PA-RNAP) to transduce polypeptide cleavage events into changes in gene expression that support phage propagation during PACE. We validate that this system successfully links the phage life cycle to protease activity for three distinct proteases. When performed in the presence of danoprevir or asunaprevir, two hepatitis $\mathrm{C}$ virus (HCV) protease inhibitor drug candidates currently in clinical trials, protease PACE rapidly evolved HCV protease variants that are resistant to each drug candidate. The PACE-evolved HCV protease variants are dominated by mutations previously observed in patients treated with these drug candidates. Together, these findings establish a new platform to rapidly generate proteases with novel properties through continuous evolution and to reveal the vulnerability of protease inhibitors to the evolution of drug resistance.

\section{Results}

Transducing protease activity into gene expression. PACE requires that a target activity be linked to changes in the expression of an essential phage gene such as gene III (gIII). To couple the cleavage of a polypeptide substrate to increases in gene expression, we engineered a PA-RNAP that transduces proteolytic activity into changes in gene expression that are sufficiently strong and rapid to support PACE. T7 RNA polymerase (T7 RNAP) is naturally inhibited when bound to T7 lysozyme ${ }^{31}$. We envisioned that T7 lysozyme could be tethered to T7 RNAP through a flexible linker containing a target protease cleavage site. Ideally, the effective concentration of the tethered T7 lysozyme with respect to T7 RNAP would be sufficiently high that the T7 RNAP subunit would exist predominantly in the T7 lysozymebound, RNAP-inactive state. Proteolysis of the target sequence would disfavour the bound T7 RNAP:T7 lysozyme complex, resulting in the liberation of an active T7 RNAP and expression of gIII placed downstream of a T7 promoter (Fig. 1a).

N-terminal fusions to T7 RNAP are known to be well tolerated, and, in the crystal structure of T7 RNAP bound to T7 lysozyme, the $\mathrm{C}$ terminus of $\mathrm{T} 7$ lysozyme is only $32 \AA$ from the $\mathrm{N}$ terminus of T7 RNAP, separated by a solvent-exposed channel ${ }^{32}$ (Supplementary Fig. 1). In light of this structural information, we linked the two proteins through these proximal termini. As T7 lysozyme activity is toxic to host $E$. coli cells, we characterized catalytically inactive lysozyme variants and found that the inactive C131S lysozyme mutant retained its ability to inhibit T7 RNAP without impairing host cell viability.

To identify T7 RNAP-T7 lysozyme linkers that promote complex formation and result in an inactive polymerase subunit yet permit efficient proteolysis, we screened a small set of linkers consisting of Gly, Ser and Ala ranging in length from 3 to 10 residues flanking each side of a target protease substrate. We designed PA-RNAP constructs containing linker peptide sequences known to be cleaved by tobacco etch virus (TEV) protease, HCV protease or human rhinovirus-14 3C (HRV) protease. We assayed T7 RNAP activity using a luciferase reporter and observed that T7 lysozyme linked to T7 RNAP through at least 28 residues including the target protease substrate resulted in significant inhibition of RNAP activity (Fig. 1b). To assay RNAP activation, we coexpressed each PA-RNAP variant from a plasmid (the complementary plasmid or CP, Fig. 1c and Supplementary Fig. 2) together with each of the three proteases (expressed from the expression plasmid or EP, Supplementary Fig. 3) in E. coli cells that also harboured a plasmid encoding gIII and luciferase under control of the T7 promoter (the accessory plasmid or AP, Fig. 1c and Supplementary Fig. 4).

Expression of a protease that is not known to cleave the target amino-acid sequence in a coexpressed PA-RNAP did not result in enhanced gene expression as measured by luciferase activity (Fig. 1d). In contrast, expression of a protease that is known to cleave the target sequence within the PA-RNAP resulted in 18- to 


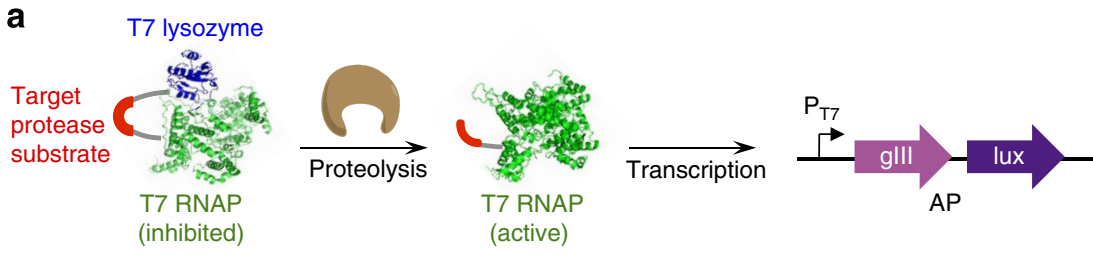

Protease-activated RNAP

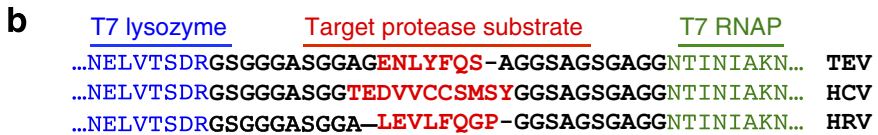

C

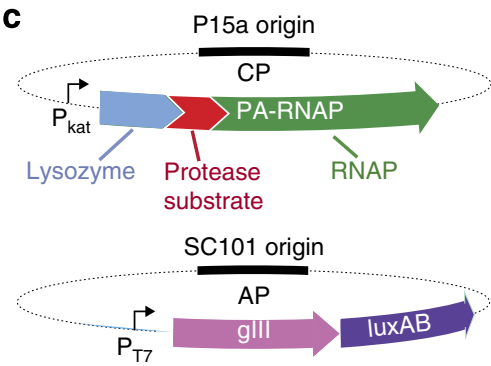

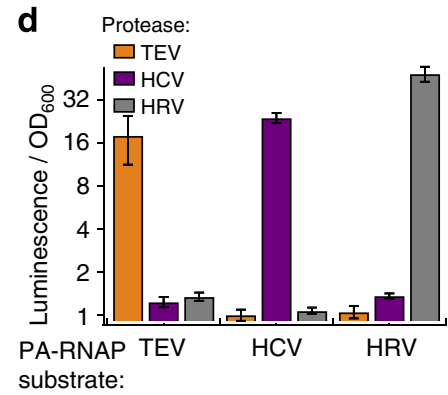

Figure 1 | Development of a system to link protease activity to gene expression. (a) Protease-activated RNA polymerase (PA-RNAP). T7 RNAP is fused to the natural inhibitor T7 lysozyme through a linker containing a protease target substrate sequence. While the linker is intact, the complex preferentially adopts the lysozyme-bound, RNAP-inactive state. Proteolysis of the target sequence favours dissociation of the complex, freeing active T7 RNAP to transcribe genes downstream of the T7 promoter. This study used an accessory plasmid (AP) in which the T7 promoter drives a tandem gIII-luciferase (lux) cassette. (b) Sequences of the protein linkers containing a target protease substrate used for each PA-RNAP, with T7 lysozyme residues in blue, protease substrates in red, T7 RNAP residues in green and linker regions in black. (c) Plasmids used for protease PACE. An AP that has gIII and luciferase (lux) under the control of the T7 promoter serves as the source of glll in the cells. A complementary plasmid (CP) constitutively expresses a PA-RNAP variant with a protease target substrate sequence embedded in the linker. (d) PA-RNAP gene expression response in E. coli cells. Host cells were transformed with (i) an AP containing the T7 promoter driving glll-lux; (ii) a CP that constitutively expresses a PA-RNAP including the TEV protease substrate, the HCV protease substrate, or the HRV protease substrate; and (iii) a plasmid that expresses TEV protease (orange bars), HCV protease (purple bars), or HRV protease (grey bars). Gene expression is activated only when the expressed protease cleaves the amino-acid sequence on the PA-RNAP sensor. The luminescence experiment was performed in triplicate with error bars indicating the s.d.

49-fold increase in gene expression for all three cognate combinations of protease and substrate. These data indicate that PA-RNAPs are capable of transducing specific proteolytic cleavage activities into large changes in target gene expression.

Linking protease activity to phage propagation. Next we sought to use PA-RNAPs to link the life cycle of M13 bacteriophage to protease activity. We generated selection phage (SP) in which gIII was replaced by a gene encoding TEV protease, HCV protease or HRV protease (Supplementary Fig. 5). Without pIII, these phage are unable to propagate on wild-type $E$. coli cells. We engineered host $E$. coli cells containing two plasmids: $(i)$ an AP that contains gIII and luciferase under the control of the T7 promoter and (ii) a CP that constitutively expresses a PA-RNAP (Fig. 1c). To be sure that the PA-RNAP selection scheme work as intended we analysed the cleavage of the sensor by western blot. We observed the loss of the lysozyme-RNAP fusion and the formation of a new protein that corresponds to the size of T7 RNAP exclusively in the presence of protease phage that recognizes the host encoded PA-RNAP (Supplementary Fig. 6). To assay whether the host cells could support phage propagation in a protease-dependent manner, we performed activity-dependent plaque assays. We observed that plaque formation, a consequence of phage replication in solid media, only occurred with phage encoding a protease that can cleave the PA-RNAP within the host cells. Phage with mismatched protease/PA-RNAP combinations did not form plaques, indicating that phage encoding non-cognate proteases do not replicate, or replicate at a significantly reduced rate. These observations together establish that the PA-RNAP system is capable of transducing protease activity of a phageencoded protease into phage production.

We next tested whether the PA-RNAP-based selection supports the continuous propagation of phage encoding active proteases in the continuous liquid culture format required for PACE (Fig. 2a). We maintained three host cell cultures, each harbouring a CP expressing a PA-RNAP containing one of the three protease cleavage sites (TEV, HCV or HRV protease substrates), using chemostats diluted with fresh growth media at a fixed rate ${ }^{30}$. Each of these host cell cultures continuously diluted lagoons seeded with various combinations of phage containing TEV, HCV or HRV protease. Lagoons seeded with phage encoding cognate proteases that can cleave the PA-RNAP within the host cells robustly propagated $\left(10^{8}-10^{10}\right.$ p.f.u. $\mathrm{ml}^{-1}$ after $72 \mathrm{~h}$ of continuous dilution at 1.0 lagoon volume per hour), while lagoons seeded with phage encoding proteases that do not match the PA-RNAP of incoming host cells washed out $\left(<10^{4}\right.$ p.f.u. $\left.\mathrm{ml}^{-1}\right)$, demonstrating protease activity-dependent propagation in continuous liquid culture.

To determine whether this system can selectively replicate phage carrying protease genes with a desired activity at the expense of phage encoding proteases that are unable to cleave the host-cell PA-RNAP, we performed protease phage enrichment experiments in a PACE format. We seeded a lagoon with a 1,000:1 ratio of TEV SP:HCV SP, then allowed the phage to propagate in the lagoon while being continuously diluted with 
a

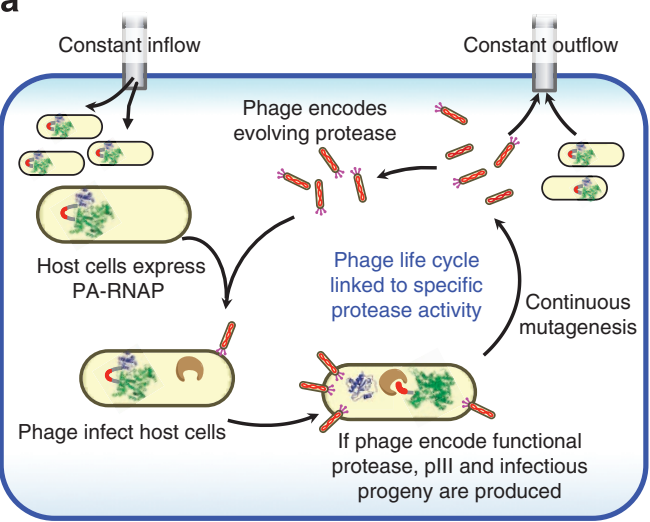

b

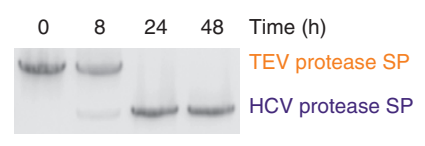

C

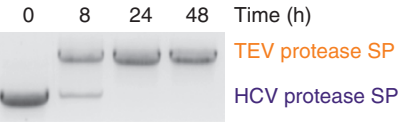

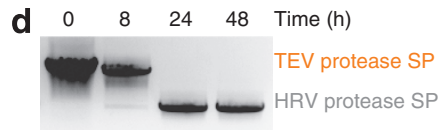

Figure 2 | PA-RNAPs link protease activity to phage propagation. (a) The protease PACE system. Fixed volume vessels (lagoons) contain phage in which gIII is replaced with a gene encoding an evolving protease. The lagoon is fed with host cells that contain an AP with the T7 promoter driving gIII and a CP that expresses a PA-RNAP. Phages infect incoming cells and inject their genome containing a protease variant. Only if the protease variant can activate the PA-RNAP by cleaving the linker encoding the target protease substrate, gIII is expressed and that SP can propagate. (b-d) Enrichment of active proteases from mixed populations using PACE. At time 0, a lagoon was seeded with a 1,000-fold excess of non-cognate protease-encoding phage over cognate protease-encoding phage. The lagoon was continuously diluted with host cells containing a PA-RNAP with either the HCV (b), TEV (c) or HRV (d) protease substrates. Lagoon samples were periodically analysed by PCR. In all three cases, phage encoding the cognate protease were rapidly enriched in the lagoon while phage encoding the non-cognate protease were depleted. Full gels are shown in Supplementary Fig. 8.

a
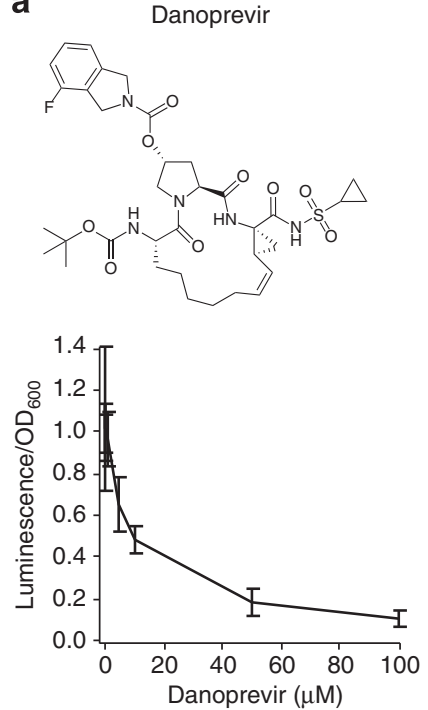

Figure 3 | HCV PA-RNAP response to protease inhibitors in E. coli cells. Host cells expressing the HCV PA-RNAP were incubated with HCV protease inhibitors danoprevir (a) or asunaprevir (b) for $90 \mathrm{~min}$, followed by inoculation with $\mathrm{HCV}$ protease encoding phage. After $3 \mathrm{~h}$, luminescence assays were used to quantify relative gene activation resulting from the PARNAP. Luminescence experiments were performed in triplicate with error bars depicting the s.d.

host cells containing a PA-RNAP with the HCV protease recognition site. We periodically sampled the waste line of the lagoon and amplified by PCR the region of the phage containing the protease genes. The TEV protease and HCV protease genes are readily distinguishable as PCR amplicons of distinct lengths. At the start of the experiment, the HCV protease phage were virtually undetectable by PCR amplification of the starting population and gel electrophoresis, while TEV protease dominated the lagoon (Fig. 2b). After just $24 \mathrm{~h}$ of continuous propagation on host cells containing the HCV PA-RNAP, the TEV protease SPs were undetectable, while the HCV protease SPs were strongly enriched ( $\geq 100,000$-fold enrichment over $24 \mathrm{~h}$ ).
We repeated this experiment with a 1,000-fold excess of HCV protease phage over TEV protease phage using host cells containing the TEV protease PA-RNAP (Fig. 2c), and a third time using a 1,000-fold excess of TEV protease phage over HRV phage and host cells containing the HRV protease PA-RNAP (Fig. 2d). In all three of the enrichment experiments, continuous propagation rapidly and markedly enriched phage encoding each cognate protease from a minute fraction of the starting phage mixture, while non-cognate proteases washed out of the lagoon (Fig. 2). Collectively, these results indicate that this protease PACE system successfully links specific protease activity to the phage life cycle in a continuous flow format and can strongly and rapidly enrich phage that encodes proteases with the ability to cleave a target polypeptide substrate.

Continuous evolution of resistance to $\mathrm{HCV}$ protease inhibitors. As an initial application of protease PACE, we continuously evolved protease enzymes to rapidly assess the drug resistance susceptibility of small-molecule protease inhibitors. Several HCV protease inhibitors are in late-stage clinical trials or are awaiting FDA approval ${ }^{33,34}$. For some HCV protease inhibitor drug candidates, clinically isolated drug-resistant mutations are known ${ }^{20}$. First we tested whether small-molecule $\mathrm{HCV}$ protease inhibitors can modulate protease activity in the protease PACE system. We observed that the incubation of host cells with either danoprevir $\left(\mathrm{IC}_{50}=\sim 0.3 \mathrm{nM}\right)^{35}$ or asunaprevir $\left(\mathrm{IC}_{50}=\sim 1.0 \mathrm{nM}\right)^{36}$, two second-generation $\mathrm{HCV}$ protease inhibitors, inhibited the cellular gene expression arising from the activity of HCV protease on the HCV PA-RNAP in a dosedependent manner (Fig. 3). These observations suggest that protease inhibitors can create selection pressure during PACE favouring the evolution of protease mutants that retain their ability to cleave a cognate substrate despite the presence of the drug candidates.

On the basis of the relationship between protease inhibitor concentration and gene expression in our system (Fig. 3) and initial trial PACE experiments, we selected $20 \mu \mathrm{M}$ danoprevir as the final concentration to use in the culture media during attempts to continuously evolve drug-resistant HCV proteases. We inoculated two separate lagoons with HCV protease SP and propagated the phage on host cells containing the HCV protease 
PA-RNAP in the absence of any inhibitor for $6 \mathrm{~h}$ to allow the accumulation of mutations in HCV protease genes. Next, we added $20 \mu \mathrm{M}$ danoprevir to the media that feeds into the host cell culture, and eventually into each of the two replicate lagoons. As a control, we propagated two replicate lagoons of HCV protease phage on HCV protease PA-RNAP host cells with no added protease inhibitor for the same time period. Throughout all of these experiments, we induced enhanced mutagenesis of the phage genome by activating an improved mutagenesis plasmid (MP) in the host cells with $0.5 \%$ arabinose (Supplementary Table 1).

Phage populations at 6 and $28 \mathrm{~h}$ from replicate lagoons were analysed by high-throughput DNA sequencing. No mutations were substantially enriched in the control lagoons propagated in the absence of any drug candidate (Fig. 4c). In contrast, several mutations rapidly evolved in both replicate lagoons in the presence of danoprevir. Mutations at position D168 were predominant among these mutations. By $28 \mathrm{~h}$, lagoon 1 with danoprevir contained 38.8\% D168E, 8.3\% D168Y, 2.1\% D168A and $1.1 \% \mathrm{D} 168 \mathrm{~V}$, while lagoon 2 with danoprevir contained $40.3 \%$ D168E and $10.7 \%$ D168Y (Fig. 4c). Other genetic differences between the SPs of these two replicate populations, such as R130C (5.1\% in lagoon 1, undetectable in lagoon 2) and
T72I (10.8\% in lagoon 2 , undetectable in lagoon 1$)$, suggest that cross-contamination did not lead to the observed protease variants in these experiments. These findings reveal that the presence of danoprevir caused the population of continuously evolving proteases to rapidly acquire mutations at D168.

To assay whether the PACE-evolved mutations confer danoprevir drug resistance in $\mathrm{HCV}$ protease, we purified recombinant $\mathrm{HCV}$ protease variants containing either of the two most highly enriched mutations, D168E and D168Y. Each of these two mutations increase the $\mathrm{IC}_{50}$ of danoprevir by $\sim 30$-fold (wild-type $\mathrm{HCV}$ protease $\mathrm{IC}_{50}=1.3 \pm 0.1 \mathrm{nM} ; \mathrm{HCV}$ protease $\mathrm{D} 168 \mathrm{E} \quad \mathrm{IC}_{50}=38.9 \pm 2.4 \mathrm{nM}$; HCV protease $\mathrm{D} 168 \mathrm{Y}$ $\mathrm{IC}_{50}=34.4 \pm 2.8 \mathrm{nM} ; \quad \mathrm{IC}_{50} \pm$ s.d.) (Fig. $4 \mathrm{~d}$ ). Importantly, the D168E, D168A and D168V mutations emerging from protease PACE have been previously identified as common drug-resistant mutations in $\mathrm{HCV}$ isolated from patients treated with danoprevir ${ }^{20,37}$.

To validate that protease PACE in the presence of a different $\mathrm{HCV}$ protease inhibitor can also result in the rapid evolution of drug-resistant mutations, we repeated PACE of HCV protease in the presence of asunaprevir, an HCV protease inhibitor in phase III clinical trials, instead of danoprevir. We selected $75 \mu \mathrm{M}$ asunaprevir as the final target concentration to use in the culture
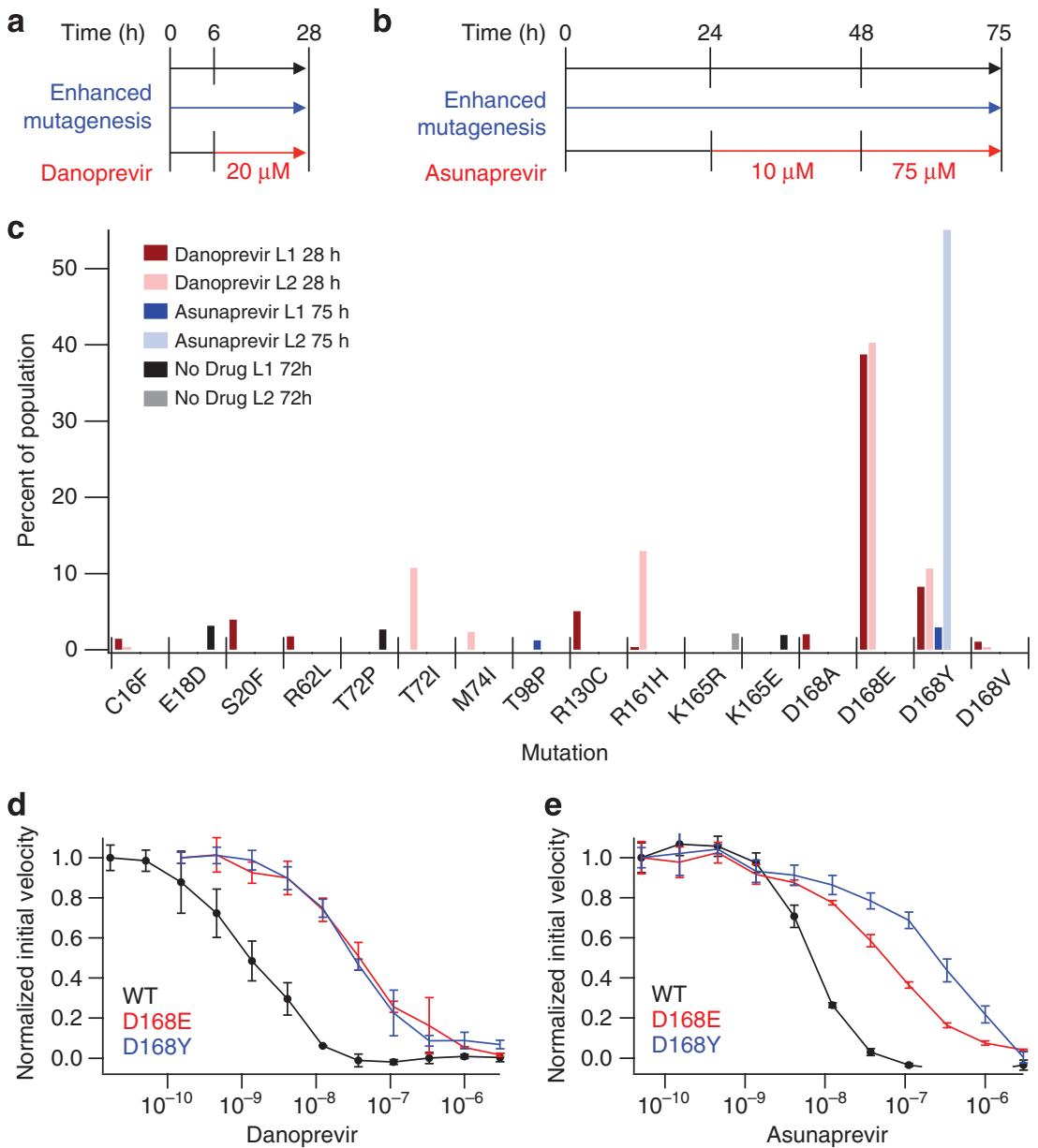

Figure 4 | Continuous evolution of drug resistance in HCV protease. (a,b) PACE condition timeline for evolution in the presence of danoprevir (a) or asunaprevir (b). The blue arrows indicate arabinose-induced enhanced mutagenesis, and the red arrow shows the timing and dosing of HCV protease inhibitors. (c) High-throughput sequencing data from phage populations in replicate lagoons (L1 and L2) subjected to danoprevir treatment at $28 \mathrm{~h}$, asunaprevir treatment at $75 \mathrm{~h}$, and no drug at $72 \mathrm{~h}$. All mutations with frequencies $>1 \%$ above the allele-specific error rate are shown. (d) In vitro analysis of danoprevir inhibition of mutant HCV proteases that evolved during PACE. (e) In vitro analysis of asunaprevir inhibition of mutant HCV proteases that evolved during PACE. For (d,e), evolved HCV protease variants were expressed and purified, then assayed using an internally quenched fluorescent-substrate (Anaspec). In vitro analyses were performed in triplicate with error bars calculated as the s.d. 
media based on dose-dependent gene expression assays (Fig. 3). To allow diversity to emerge in the protease population, we first propagated HCV protease phage for $24 \mathrm{~h}$ without any inhibitor. Next, to ensure that the populations had sufficient time to evolve mutations that confer drug resistance, we propagated the populations for $24 \mathrm{~h}$ with $10 \mu \mathrm{M}$ asunaprevir, Finally, we ramped up the asunaprevir concentration to $75 \mu \mathrm{M}$ for $27 \mathrm{~h}$ to enrich those mutations that conferred robust drug resistance. HCV protease phage were also propagated for an identical amount of time without any added drug candidate for comparison. Highthroughput DNA sequencing of phage populations at the end of the experiment revealed that mutations evolved at substantial levels in the asunaprevir-treated lagoons but not in the control samples (Fig. 4c). In this experimental condition as well, mutations at position D168 were highly enriched. In the case of asunaprevir, however, the only substitution at this position to emerge at substantial levels from protease PACE was D168Y, in contrast with the evolution of both D168E and D168Y during protease PACE with danoprevir.

In vitro assays of $\mathrm{HCV}$ proteases containing either mutation provides an explanation underlying the strong apparent preference of D168Y over D168E within asunaprevir-treated lagoons. D168Y increases the $\mathrm{IC}_{50}$ of asunaprevir by 30 -fold, while D168E only increases the $\mathrm{IC}_{50}$ of asunaprevir by $\sim 10$-fold (wild-type $\mathrm{HCV}$ protease $\mathrm{IC}_{50}=6.9 \pm 0.6 \mathrm{nM}$; HCV protease $\mathrm{D} 168 \mathrm{E}$ $\mathrm{IC}_{50}=53.5 \pm 3.4 \mathrm{nM} ; \mathrm{HCV}$ protease D168Y $\mathrm{IC}_{50}=214.8 \pm 31.9$ $\mathrm{nM} ; \mathrm{IC}_{50} \pm$ s.d.) (Fig. 4e). Mutations at position D168 have been previously identified in replicon-based asunaprevir resistance experiments $^{38}$, and the specific D168Y mutation has been observed to arise in hepatitis $\mathrm{C}$ patients treated with asunaprevir $^{39}$. Collectively, these results establish that protease PACE in the presence of protease inhibitor drug candidates can very rapidly (1-3 days) reveal clinically relevant mutants that confer strong resistance to the drug candidates, without requiring extensive laboratory or clinical experiments.

\section{Discussion}

Previous efforts to use laboratory evolution to study $\mathrm{HCV}$ protease inhibitor resistance have relied on time- and laborintensive approaches such as viral replication in mammalian cell culture or conventional protein evolution methods, which typically require months to complete ${ }^{22}$. By comparison, the continuous evolution of proteases can reveal key resistance mutations in as little as $\sim 1$ day of PACE. The speed of PACE and its ability to be multiplexed using many lagoons in parallel, each receiving a different drug candidate, and analysed by highthroughput DNA sequencing of bar-coded lagoon samples, raises the possibility of screening future early-stage hit or lead compounds for their vulnerability to the evolution of drug resistance, before more resource-intensive optimization of in vivo properties or clinical trials take place. Rapid and cost-effective access to drug resistance susceptibility enabled by PACE may enhance the more informed selection of more promising earlystage drug candidates for further development. This technique could also be applied to quickly screen a drug candidate across many distinct genotypic variants of a protease target (such as the six major HCV protease genotypes) to reveal each target variant's potential to evolve mutations that abrogate the effectiveness of the drug candidate. As HCV patient isolates and replicon assays have already demonstrated differing drug resistance profiles among different HCV genotypes ${ }^{40}$, this capability could also be used to rapidly identify patient-specific drug treatments that are more likely to offer long-term therapeutic effects on patients infected with specific HCV strains, even in the absence of previous data relating strain genotypes to drug effectiveness.
The development of protease PACE also expands the scope of PACE to evolve diverse biochemical activities. Previous PACE studies have only evolved RNA polymerases, which have activities that can be directly linked to changes in gIII expression. This study demonstrates how other types of enzymatic activities with no obvious direct connection to gene expression can nevertheless be evolved using PACE by establishing an indirect, but robust, linkage between the activity of interest and gIII expression.

The protease PACE system provides a strong foundation for the continuous evolution of proteases with reprogrammed specificities. Previous work on reprogramming the DNA substrate selectivity of T7 RNAP enzymes ${ }^{25,28-30}$ demonstrated the ability of PACE to rapidly evolve enzymes that accept substrates very different from the native substrate. These reprogramming experiments relied on a 'stepping-stone' strategy in which SPs are transitioned between a series of intermediate substrates ${ }^{28}$, and are enhanced by the recent development of modulated selection stringency and negative selection during $\mathrm{PACE}^{30}$. In principle these strategies coupled with protease PACE should also enable the continuous evolution of protease enzymes with the tailormade ability to selectively cleave proteins implicated in human diseases.

\section{Methods}

PA-RNAP gene expression response in vivo. All plasmids were constructed by Gibson Assembly 2x Master Mix (NEB); all PCR products were generated using Q5 Hot Start 2x Master Mix (NEB). E. coli strain S1030 (ref. 30) were transformed by electroporation with three plasmids: $(i)$ a complementary plasmid $(\mathrm{CP})$ that constitutively expresses a PA-RNAP with one of the three protease cut sites (Supplementary Fig. 2), (ii) an accessory plasmid (AP, Supplementary Fig. 4) that encodes gIII-luciferase (translationally coupled) under control of the T7 promoter and (iii) an arabinose-inducible expression plasmid for one of the three proteases (EP, Supplementary Fig. 3). The HRV protease gene was purchased as IDT gblocks and cloned into the expression vector. The MBP-TEV fusion protein was amplified by PCR from pRK793 (ref. 41). The MBP fusion was necessary for expression and solubility. We deployed a constitutively active HCV protease construct that includes the NS4a cofactor peptide ${ }^{42}$. Cells were grown in $2 \mathrm{xYT}$ media to saturation in the presence of antibiotics and $1 \mathrm{mM}$ glucose, then inoculated into $1 \mathrm{ml}$ fresh media containing $1 \mathrm{mM}$ glucose and antibiotics in a 96-well culture plate. After $4.5 \mathrm{~h}, 150 \mu \mathrm{l}$ of the cultures were transferred to a black-wall clearbottom assay plate and luciferase, and $\mathrm{OD}_{600}$ measurements were taken using a Tecan Infinite Pro plate. The luminescence data were normalized to cell density by dividing by $\mathrm{OD}_{600}$.

Western blot of PA-RNAP sensor activation. E. coli cells transformed with an $\mathrm{AP}$ and a CP were grown to log phase, then infected with a 10 -fold excess of protease-encoding phage. After $4.5 \mathrm{~h}$, the cells were harvested by centrifugation at $5,000 \mathrm{~g}$ for $10 \mathrm{~min}$, and then resuspended in LDS Sample Buffer (Life Technologies). Samples were heated to $95^{\circ} \mathrm{C}$ for $5 \mathrm{~min}$ and vortexed to shear genomic DNA. Four microlitres of each sample was loaded onto a protein gel electrophoresis system (Bolt gel system, Life Technologies). The blot was performed using a PVDF membrane (iBlot 2 system, Life Technologies). The membrane was blocked with $5 \%$ BSA TBST then incubated overnight with the primary antibody (5\% BSA, TBST, 1:5,000 anti-T7 RNAP mouse monoclonal, Novagen \#70566). The membrane was washed three times, incubated with the secondary antibody (5\% BSA, TBST, 1:5,000 donkey anti-mouse, IR-dye conjugate, LI-COR \#926-32212) for $60 \mathrm{~min}$, washed three times, then visualized on a LI-COR Odyssey at $800 \mathrm{~nm}$. As seen in Supplementary Fig. 6, the PA-RNAP sensor is proteolysed to a smaller band of anticipated molecular weight only in the presence of a cognate protease that can cleave the peptide sequence in each PA-RNAP linker.

Protease activity-dependent plaque assays. Protease phages were cloned using Gibson assembly and the aforementioned expression plasmids as templates. E. coli strain S1030 was transformed by electroporation with an AP and a CP. After the transformed host cells were grown in $2 \mathrm{xYT}$ to $\mathrm{OD}_{600} \sim 1.0,100 \mu \mathrm{l}$ of cells was added to $50 \mu \mathrm{l}$ of serial dilutions of protease-encoding phage. After $1 \mathrm{~min}, 800 \mu \mathrm{l}$ of top agar $\left(7 \mathrm{gl}^{-1}\right.$ agar in $\left.2 \mathrm{xYT}\right)$ was added, mixed and transferred to quarter-plates containing bottom agar $\left(15 \mathrm{gl}^{-1}\right.$ agar in $\left.2 \mathrm{xYT}\right)$. After overnight incubation at $37^{\circ} \mathrm{C}$, the plates were examined for plaques, which represent zones of slowed growth and diminished turbidity due to phage propagation.

PACE propagations and enrichment experiments. E. coli strain S1030 was transformed by electroporation with an AP, CP (one for each of the three PA-RNAPs) and a MP (Supplementary Fig. 7, Supplementary Table 1) encoding 
arabinose-inducible expression of a dominant-negative mutator variant of $d n a Q$, wild-type dam and wild-type seq $A^{43-45}$. Starter cultures were grown overnight in $2 \mathrm{xYT}$ supplemented with antibiotics and $1 \mathrm{mM}$ glucose to prevent induction of mutagenesis before the PACE experiment. Host cell culture chemostats containing $80 \mathrm{ml}$ of Davis rich media ${ }^{30}$ were inoculated with $2 \mathrm{ml}$ of starter culture and grown at $37^{\circ} \mathrm{C}$ with magnetic stir-bar agitation. At approximately $\mathrm{OD}_{600} 1.0$, fresh Davis rich media was pumped in at $80-100 \mathrm{ml} \mathrm{h}^{-1}$, with a chemostat waste needle set at $80 \mathrm{ml}$. This fixed dilution rate maintains the chemostat culture in late log phase growth, at which point it can be flowed into lagoons seeded with protease phage (initial titers were $\sim 10^{5}$ p.f.u. $\mathrm{ml}^{-1}$ ). For these experiments, lagoon waste needles were set to maintain a lagoon volume of $15 \mathrm{ml}$, and host cell cultures were flowed in at $15-17 \mathrm{ml} \mathrm{h}^{-1}$. Arabinose $(10 \% \mathrm{w} / \mathrm{v}$ in water) was added directly to lagoons via syringe pump at $0.7 \mathrm{mlh}^{-1}$ to induce mutagenesis. Test propagations were conducted with cognate protease phage as well as non-cognate protease phage. Enrichment experiment lagoons were seeded with 1,000-fold excess of non-cognate protease phage. Lagoon samples were sterile-filtered at least every $24 \mathrm{~h}$, and titres were assessed by plaque assay. Plaque assays were performed with S1030 carrying pJC175e, a plasmid that supplies gIII under control of the phage-shock promoter ${ }^{30}$. Mock selections were monitored by PCR of the protease genes with forward primer

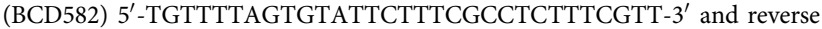
primer (BCD578) 5'-CCCACAAGAATTGAGTTAAGCCCAATAATAAGAGC- $3^{\prime}$ using filtered samples as templates. The distinct sizes of amplicons containing protease genes enabled evaluation of the relative abundance of cognate and non-cognate protease-encoding phage. Uncropped gel images are shown in Supplementary Fig. 8.

Inhibition of PA-RNAP response in host $\boldsymbol{E}$. coli cells. Host cells were prepared by electroporation with an AP and the CP encoding the HCV-site PA-RNAP. We prepared $2 \mathrm{xYT}$ media with serial dilutions of inhibitors (danoprevir and asunaprevir, MedChemExpress) from stock solutions made in DMSO and inoculated with a saturated starter culture of host cells. $150 \mu \mathrm{l}$ cell cultures in a 96-well assay plate were incubated at $37^{\circ} \mathrm{C}$ for $1.5 \mathrm{~h}$ to allow uptake of inhibitors, then infected with $\sim 10 \mu \mathrm{l} \mathrm{HCV}$ protease phage (multiplicity of infection $\sim 10$ ). After $3 \mathrm{~h}$ of incubation at $37^{\circ} \mathrm{C}$, the luminescence of each culture was measured on a Tecan Infinite Pro plate reader and normalized to $\mathrm{OD}_{600}$. In the absence of inhibitor, phage-encoded protease will activate the PA-RNAP leading to robust production of luciferase. Relative dose responses to inhibitors compared with control cells without drug were measured in triplicate.

Improved mutagenesis plasmid. The previous generation of the $\mathrm{MP}^{25}$ carried four genes: dnaQ926 (ref. 44) (a dominant-negative E. coli DNA polymerase III proofreading subunit), $u m u D^{\prime}$ and $u m u C$ (the components of $E$. coli translesion synthesis polymerase $\mathrm{V}$ ) and recA730 (an activated $r e c A$ mutant). The complex of $\mathrm{UmuD}_{2}{ }_{2} \mathrm{C} / \mathrm{Rec} \mathrm{A} 730$ forms the $E$. coli mutasome complex, a critical requirement for translesion synthesis across predominantly T-T (6-4) photoproducts and pyrimidine dimers ${ }^{46,47}$. As neither type of mutation is predicted to occur commonly during our current PACE experiments, which do not use UV light or chemical mutagens, the genes encoding the mutasome were removed from the MP. To improve the efficiency of mutagenesis, two additional proteins were included on the $P_{B A D}$ transcript: dam (deoxyadenosine methylase) and seqA (a hemimethylated-GATC binding domain), both of which are known mutators when overexpressed in $E$. coli $i^{43,45}$. The combination of these three genes yielded higher mutagenesis rates in the presence of arabinose during PACE and resulted in fivefold higher mutagenesis of E. coli chromosomal DNA as assessed by a rifampin resistance assay (Supplementary Table 1).

Rifampin resistance assay. MG1655 $\Delta$ recA E. coli ${ }^{48}$ (CGSC\#: 12492) cells were transformed with the appropriate MPs and plated on 2xYT/agar plates supplemented with $40 \mathrm{\mu g} \mathrm{ml}^{-1}$ chloramphenicol and $100 \mathrm{mM}$ glucose to ensure that no induction occurs before the assay. After overnight growth, single colonies were picked into liquid Davis Rich Media ${ }^{49}$ supplemented with $40 \mu \mathrm{g} \mathrm{ml}^{-1}$ chloramphenicol and grown for $12-16 \mathrm{~h}$ with vigorous shaking at $37^{\circ} \mathrm{C}$. Cultures were then diluted 1,000-fold in Davis Rich Media and grown until they reached $\mathrm{OD}_{600}=0.5-0.7$, at which point they were split into two equal volumes, supplemented with either $100 \mathrm{mM}$ glucose or $100 \mathrm{mM}$ arabinose, and allowed to grow for an additional $24 \mathrm{~h}$. Saturated cultures were serially diluted and plated on 2xYT/agar supplemented with $100 \mathrm{mM}$ glucose with or without $100 \mu \mathrm{g} \mathrm{ml}^{-1}$ rifampin. After overnight growth, colonies were counted from both plates, and the frequency of resistant mutants was calculated. This measurement been widely used in the literature as a metric of mutagenesis ${ }^{50}$.

Evolution of drug resistance in HCV protease using PACE. Host cells were the same as those in the HCV test propagation and enrichment experiments. Host cell culture chemostats containing $40 \mathrm{ml}$ of Davis rich media were inoculated with $2 \mathrm{ml}$ of starter culture and grown at $37^{\circ} \mathrm{C}$ with magnetic stir-bar agitation. At approximately $\mathrm{OD}_{600} 1.0$, fresh Davis rich media was pumped in at $50 \mathrm{ml} \mathrm{h}^{-1}$, with a chemostat waste (outflow) needle set at $40 \mathrm{ml}$. This adjustment was made to provide enough cell culture to feed two lagoons while also conserving media that contained small-molecule inhibitors. Lagoons were seeded with HCV protease phage and run in duplicate. Again, lagoon waste needles were set to maintain a lagoon volume of $15 \mathrm{ml}$, and host cell cultures were flowed in at $15-17 \mathrm{ml} \mathrm{h}^{-1}$. Arabinose $(10 \% \mathrm{w} / \mathrm{v}$ in water) was added directly to lagoons via syringe pump at $0.7 \mathrm{ml} \mathrm{h}^{-1}$ to induce the expression of mutagenesis genes on the MP.

After $6 \mathrm{~h}$ of propagation without any inhibitor, a filtered lagoon sample was taken, and danoprevir was added directly to the chemostat media at $20 \mu \mathrm{M}$ with $2.5 \%$ DMSO to enhance solubility. A final time point was taken after 22 additional hours, and titres were measured by plaque assay on strain S1030 carrying pJC175e

For the asunaprevir experiment, samples were taken every $12 \mathrm{~h}$. After $24 \mathrm{~h}$ of propagation with no inhibitor, asunaprevir was added directly to the chemostat media at $10 \mu \mathrm{M}$ with $2.5 \%$ DMSO. After an additional $24 \mathrm{~h}$, asunaprevir dosage was increased to $75 \mu \mathrm{M}$ and $5 \%$ DMSO. Titres were measured by plaque assay on strain S1030 carrying pJC175e.

High-throughput sequencing of evolved populations. Strain S1030 carrying pJC175e was grown to saturation and used to inoculate fresh media. Host cells were infected with phage samples from the above PACE experiments and incubated for $5 \mathrm{~h}$ at $37^{\circ} \mathrm{C}$. DNA from infected cells was extracted using miniprep kits to yield concentrated template phage DNA (Epoch Life Science). PCR reactions were performed using Q5 Hot Start 2x Master Mix (NEB) with a set of tiled primers. The PCR product from the first reaction was diluted ten-fold and $1 \mu \mathrm{l}$ served as the template for the second PCR. The second PCR added Illumina adapters as well as barcodes; PCR products were purified from agarose gel (Qiagen) and quantified using the Quant-IT Picogreen assay (Invitrogen). Samples were normalized and pooled together to create a sequencing library at $\sim 4 \mathrm{nM}$. The library was quantified by qPCR (KapaBiosystems) and processed by an Illumina MiSeq using the MiSeq Reagent Kit v3 and the $2 \times 300$ paired-end protocol. A single paired-end read of $600 \mathrm{bp}$ is sufficient to cover the entire HCV protease gene.

Data were analysed in MATLAB using the custom scripts supplied in the Supplementary Software. FASTQ files were automatically generated by the Illumina MiSeq. These files were already binned by sample barcodes and ready for transfer to a desktop computer. Each read was aligned to the wild-type HCV protease gene in the expected orientation using the Smith-Waterman algorithm. Base calls with Q-scores below a threshold of 31 were converted to ambiguous bases, and the resulting ambiguous codons were turned into a series of three dashes for computationally efficient translation. Ambiguous codons were translated into Xs, which were ignored when tabulating allele counts into a matrix. The script automatically cycled through each FASTQ file and saved the resulting allele count matrix in a separate subdirectory. At this stage, matrices for paired-end reads were added together and normalized to yield allele frequencies for each sample.

We relied on a wild-type control sample to assess PCR and sequencing bias. For this sample, we calculated the frequency of alleles that were not wild type at each locus to yield the locus-specific error rate. We added 0.01 (1\%) to the locus-specific error rate to yield our variant call threshold. The allele frequency matrix for each sample was scanned for mutant alleles above the variant call threshold.

\section{Purification and in vitro assays of evolved $\mathbf{H C V}$ variants. $\mathrm{HCV}$ protease} variants were subcloned by Gibson assembly out of the phage genome and into the previously mentioned EP. EPs were transformed into NEB BL21 DE3 chemically competent cells. Starter cultures were grown to saturation, and $2 \mathrm{ml}$ was used to inoculate $500 \mathrm{ml} \mathrm{LB}$. At $\mathrm{OD}_{600}=0.6$, cultures were transferred to $20^{\circ} \mathrm{C}$ and induced with $0.5 \%$ arabinose for $6 \mathrm{~h}$. Cells were harvested by centrifugation at $5,000 \mathrm{~g}$ for $10 \mathrm{~m}$, and resuspended in lysis/bind buffer (50 mM Tris- $\mathrm{HCl} \mathrm{pH} \mathrm{8.0,}$ $500 \mathrm{mM} \mathrm{NaCl}, 10 \%$ glycerol, $5 \mathrm{mM}$ imidazole). Cells were lysed by sonication for a total of $2 \mathrm{~min}$, and then centrifuged for $20 \mathrm{~min}$ at $18,000 \mathrm{~g}$ to clarify the lysate. Supernatant was flowed through $0.2 \mathrm{ml}$ His-pur nickel resin spin columns that were equilibrated with binding buffer (Pierce-Thermo). Resin was washed with four column volumes of wash buffer $(50 \mathrm{mM}$ Tris- $\mathrm{HCl} \mathrm{pH} 8.0,500 \mathrm{mM} \mathrm{NaCl}$, $10 \%$ glycerol, $20 \mathrm{mM}$ imidazole). HCV protease was eluted in four column volumes of $50 \mathrm{mM}$ Tris- $\mathrm{HCl} \mathrm{pH} 8.0,500 \mathrm{mM} \mathrm{NaCl}, 10 \%$ glycerol, $200 \mathrm{mM}$ imidazole. Samples were further purified by size exclusion chromatography on a SuperDex 75 10/300 GL column (GE Healthcare). Size exclusion was performed in $50 \mathrm{mM}$ Tris- $\mathrm{HCl} \mathrm{pH}$ 8.0, $100 \mathrm{mM} \mathrm{NaCl}, 10 \%$ glycerol, $1 \mathrm{mM}$ DTT. Protein concentrations were determined by $\mathrm{UV}_{280}$ on a Nanodrop machine and calculated using an extinction coefficient of $19,000 \mathrm{~cm}^{-1} \mathrm{M}^{-1}$ and a molecular weight of $23 \mathrm{kDa}$.

In vitro assays were performed using the commercial HCV RET Substrate 1 (Anaspec), an internally quenched probe that fluoresces on proteolytic cleavage, according to the manufacturer's instructions. Protease and inhibitors were incubated in assay buffer at room temperature for $5 \mathrm{~m}$ before addition of substrate. Fluorescence was measured every $30 \mathrm{~s}$ for $20 \mathrm{~m}$ by a Tecan Infinite Pro plate reader (excitation/emission $=355 \mathrm{~nm} / 495 \mathrm{~nm}$ ). Assays were performed at $30^{\circ} \mathrm{C}$ with $40 \mathrm{nM}$ protease, $7.5 \mu \mathrm{M}$ substrate and varying concentration of inhibitors in a final volume of $100 \mu \mathrm{l}$ per well in black-wall clear-bottom assay plate. The assay buffer contained $50 \mathrm{mM}$ Tris $\mathrm{HCl}$ pH $8.0,100 \mathrm{mM} \mathrm{NaCl}, 20 \%$ glycerol, $5 \mathrm{mM}$ DTT. Assays were performed in triplicate, and initial reaction velocities were calculated and normalized to controls without inhibitor. The data were fit to the Hill Equation using Igor Pro with base and max parameters fixed at one and zero, respectively. The resulting fits yielded $\mathrm{IC}_{50}$ values and s.d. of the estimate. 


\section{References}

1. Schilling, O. \& Overall, C. M. Proteome-derived, database-searchable peptide libraries for identifying protease cleavage sites. Nat. Biotechnol. 26, 685-694 (2008).

2. Walsh, G. Biopharmaceutical benchmarks 2006. Nat. Biotechnol. 24, 769-776 (2006).

3. Wehr, M. C. et al. Monitoring regulated protein-protein interactions using split TEV. Nat. Methods 3, 985-993 (2006).

4. Craik, C. S., Page, M. J. \& Madison, E. L. Proteases as therapeutics. Biochem. J. 435, 1-16 (2011)

5. Gray, D. C., Mahrus, S. \& Wells, J. A. Activation of specific apoptotic caspases with an engineered small-molecule-activated protease. Cell 142, 637-646 (2010).

6. von Mehren, M., Adams, G. P. \& Weiner, L. M. Monoclonal antibody therapy for cancer. Annu. Rev. Med. 54, 343-369 (2003).

7. Waldmann, T. A. Immunotherapy: past, present and future. Nat. Med. 9, 269-277 (2003).

8. Caravella, J. \& Lugovskoy, A. Design of next-generation protein therapeutics. Curr. Opin. Chem. Biol. 14, 520-528 (2010).

9. Russell, A. J. \& Fersht, A. R. Rational modification of enzyme catalysis by engineering surface charge. Nature 328, 496-500 (1987).

10. Varadarajan, N., Gam, J., Olsen, M. J., Georgiou, G. \& Iverson, B. L. Engineering of protease variants exhibiting high catalytic activity and exquisite substrate selectivity. Proc. Natl Acad. Sci. USA 102, 6855-6860 (2005)

11. Knight, Z. A., Garrison, J. L., Chan, K., King, D. S. \& Shokat, K. M. A remodelled protease that cleaves phosphotyrosine substrates. J. Am. Chem. Soc. 129, 11672-11673 (2007).

12. Sellamuthu, S. et al. Engineering of protease variants exhibiting altered substrate specificity. Biochem. Biophys. Res. Commun. 371, 122-126 (2008).

13. Varadarajan, N., Rodriguez, S., Hwang, B. Y., Georgiou, G. \& Iverson, B. L. Highly active and selective endopeptidases with programmed substrate specificities. Nat. Chem. Biol. 4, 290-294 (2008).

14. Yi, L. et al. Engineering of TEV protease variants by yeast ER sequestration screening (YESS) of combinatorial libraries. Proc. Natl Acad. Sci. USA 110, 7229-7234 (2013).

15. Abbenante, G. \& Fairlie, D. P. Protease inhibitors in the clinic. Med. Chem. 1, 71-104 (2005).

16. Turk, B. Targeting proteases: successes, failures and future prospects. Nat. Rev. Drug Discov. 5, 785-799 (2006).

17. Rong, L., Dahari, H., Ribeiro, R. M. \& Perelson, A. S. Rapid emergence of protease inhibitor resistance in hepatitis C virus. Sci. Transl. Med. 2, 30 ra32 (2010).

18. Ridky, T. \& Leis, J. Development of drug resistance to HIV-1 protease inhibitors. J. Biol. Chem. 270, 29621-29623 (1995).

19. Lefebvre, E. \& Schiffer, C. A. Resilience to resistance of HIV-1 protease inhibitors: profile of darunavir. AIDS Rev. 10, 131-142 (2008).

20. Romano, K. P. et al. The molecular basis of drug resistance against hepatitis $C$ virus NS3/4A protease inhibitors. PLoS Pathog. 8, e1002832 (2012).

21. Shang, L., Lin, K. \& Yin, Z. Resistance mutations against HCV protease inhibitors and antiviral drug design. Curr. Pharm. Des. 20, 694-703 (2014).

22. Verbinnen, T. et al. Tracking the evolution of multiple in vitro hepatitis $C$ virus replicon variants under protease inhibitor selection pressure by 454 deep sequencing. J. Virol. 84, 11124-11133 (2010).

23. Billerbeck, E., de Jong, Y., Dorner, M., de la Fuente, C. \& Ploss, A. Animal models for hepatitis C. Curr. Top. Microbiol. Immunol. 369, 49-86 (2013).

24. Orencia, M. C., Yoon, J. S., Ness, J. E., Stemmer, W. P. \& Stevens, R. C. Predicting the emergence of antibiotic resistance by directed evolution and structural analysis. Nat. Struct. Biol. 8, 238-242 (2001).

25. Esvelt, K. M., Carlson, J. C. \& Liu, D. R. A system for the continuous directed evolution of biomolecules. Nature 472, 499-503 (2011).

26. Hedstrom, L., Szilagyi, L. \& Rutter, W. J. Converting trypsin to chymotrypsin: the role of surface loops. Science 255, 1249-1253 (1992).

27. Kim, J. L. et al. Crystal structure of the hepatitis C virus NS3 protease domain complexed with a synthetic NS4A cofactor peptide. Cell 87, 343-355 (1996).

28. Dickinson, B. C., Leconte, A. M., Allen, B., Esvelt, K. M. \& Liu, D. R. Experimental interrogation of the path dependence and stochasticity of protein evolution using phage-assisted continuous evolution. Proc. Natl Acad. Sci. USA 110, 9007-9012 (2013).

29. Leconte, A. M. et al. A population-based experimental model for protein evolution: effects of mutation rate and selection stringency on evolutionary outcomes. Biochemistry 52, 1490-1499 (2013).

30. Carlson, J. C., Badran, A. H., Guggiana-Nilo, D. A. \& Liu, D. R. Negative selection and stringency modulation in phage-assisted continuous evolution. Nat. Chem. Biol. 10, 216-222 (2014).

31. Entus, R., Aufderheide, B. \& Sauro, H. M. Design and implementation of three incoherent feed-forward motif based biological concentration sensors. Syst. Synth. Biol. 1, 119-128 (2007).

32. Jeruzalmi, D. \& Steitz, T. A. Structure of T7 RNA polymerase complexed to the transcriptional inhibitor T7 lysozyme. EMBO J. 17, 4101-4113 (1998).

33. Clark, V. C., Peter, J. A. \& Nelson, D. R. New therapeutic strategies in HCV: second-generation protease inhibitors. Liver Int. 33(Suppl 1): 80-84 (2013).
34. Manns, M. P. \& von Hahn, T. Novel therapies for hepatitis C - one pill fits all? Nat. Rev. Drug Discov. 12, 595-610 (2013).

35. Jiang, Y. et al. Discovery of danoprevir (ITMN-191/R7227), a highly selective and potent inhibitor of hepatitis $\mathrm{C}$ virus (HCV) NS3/4A protease. J. Med. Chem. 57, 1753-1769 (2014).

36. Scola, P. M. et al. The discovery of asunaprevir (BMS-650032), an orally efficacious NS3 protease inhibitor for the treatment of hepatitis $\mathrm{C}$ virus infection. J. Med. Chem. 57, 1730-1752 (2014).

37. Lim, S. R. et al. Virologic escape during danoprevir (ITMN-191/RG7227) monotherapy is hepatitis C virus subtype dependent and associated with $\mathrm{R} 155 \mathrm{~K}$ substitution. Antimicrob. Agents Chemother. 56, 271-279 (2012).

38. McPhee, F. et al. Resistance analysis of the hepatitis C virus NS3 protease inhibitor asunaprevir. Antimicrob. Agents Chemother. 56, 3670-3681 (2012).

39. McPhee, F. et al. Resistance analysis of hepatitis $\mathrm{C}$ virus genotype 1 prior treatment null responders receiving daclatasvir and asunaprevir. Hepatology 58, 902-911 (2013).

40. Imhof, I. \& Simmonds, P. Genotype differences in susceptibility and resistance development of hepatitis $C$ virus to protease inhibitors telaprevir (VX-950) and danoprevir (ITMN-191). Hepatology 53, 1090-1099 (2011).

41. Kapust, R. B. et al. Tobacco etch virus protease: mechanism of autolysis and rational design of stable mutants with wild-type catalytic proficiency. Protein Eng. 14, 993-1000 (2001).

42. Romano, K. P., Ali, A., Royer, W. E. \& Schiffer, C. A. Drug resistance against HCV NS3/4A inhibitors is defined by the balance of substrate recognition versus inhibitor binding. Proc. Natl Acad. Sci. USA 107, 20986-20991 (2010).

43. Herman, G. E. \& Modrich, P. Escherichia coli K-12 clones that overproduce dam methylase are hypermutable. J. Bacteriol. 145, 644-646 (1981).

44. Fijalkowska, I. J. \& Schaaper, R. M. Mutants in the Exo I motif of Escherichia coli dnaQ: defective proofreading and inviability due to error catastrophe. Proc Natl Acad. Sci. USA 93, 2856-2861 (1996).

45. Yang, H., Wolff, E., Kim, M., Diep, A. \& Miller, J. H. Identification of mutator genes and mutational pathways in Escherichia coli using a multicopy cloning approach. Mol. Microbiol. 53, 283-295 (2004).

46. Bagg, A., Kenyon, C. J. \& Walker, G. C. Inducibility of a gene product required for UV and chemical mutagenesis in Escherichia coli. Proc. Natl Acad. Sci. USA 78, 5749-5753 (1981).

47. Burckhardt, S. E., Woodgate, R., Scheuermann, R. H. \& Echols, H. UmuD mutagenesis protein of Escherichia coli: overproduction, purification, and cleavage by RecA. Proc. Natl Acad. Sci. USA 85, 1811-1815 (1988).

48. Mahdi, A. A., Buckman, C., Harris, L. \& Lloyd, R. G. Rep and PriA helicase activities prevent RecA from provoking unnecessary recombination during replication fork repair. Genes Dev. 20, 2135-2147 (2006).

49. Carlson, J. C., Badran, A. H., Guggiana-Nilo, D. A. \& Liu, D. R. Negative selection and stringency modulation enable phage-assisted continuous evolution (PACE) of enzymes with altered specificity. Nat. Chem. Biol. 10, 216-222 Accepted (2013).

50. Cupples, C. G. \& Miller, J. H. A set of lacZ mutations in Escherichia coli that allow rapid detection of each of the six base substitutions. Proc. Natl Acad. Sci. USA 86, 5345-5349 (1989).

\section{Acknowledgements}

We thank Celia Schiffer for providing the gene for the HCV NS3 protease domain fused to the NS4a cofactor peptide. We thank Kevin Esvelt and Jacob Carlson for providing materials and helpful discussions. This research was supported by DARPA HR0011-11 2-0003, DARPA N66001-12-C-4207, and the Howard Hughes Medical Institute. B.C.D. is a Fellow of the Jane Coffin Childs Memorial Fund for Medical Research. M.S.P. is an NSF Graduate Research Fellow and was supported by the Harvard Biophysics NIH training grant NIH NIGMS T32 GM008313. A.H.B. was supported by a National Science Foundation Graduate Research Fellowship and the Harvard Chemical Biology Program.

\section{Author contributions}

B.C.D. and M.S.P. designed the research, prepared materials and performed experiments. A.H.B. developed and prepared the improved mutagenesis plasmid. D.R.L. designed and supervised the research. All of the authors contributed to the manuscript.

\section{Additional information}

Supplementary Information accompanies this paper at http://www.nature.com/ naturecommunications

Competing financial interests: The authors have filed a provisional patent application on PACE and related improvements.

Reprints and permission information is available online at http://www.nature.com/ reprintsandpermission/

How to cite this article: Dickinson, B. C. et al. A system for the continuous directed evolution of proteases rapidly reveals drug-resistant mutations. Nat. Commun. 5:5352 doi: $10.1038 /$ ncomms6352 (2014) 